\title{
Antibody to the Rheumatoid Arthritis Nuclear Antigen
}

\author{
ITS RELATIONSHIP TO IN VIVO EPSTEIN-BARR VIRUS INFECTION
}

\author{
Michael A. Catalano, Dennis A. Carson, James C. Niederman, Paul Feorino, \\ and John H. Vaughan, Department of Clinical Research, Scripps Clinic \\ and Research Foundation, La Jolla, California 92037; Department of Epidemiology \\ and Public Health, Yale University School of Medicine, New Haven, Connecticut \\ 06510; Special Viral Diagnostic Branch Center for Disease Control, Atlanta, \\ Georgia 30333
}

\begin{abstract}
A B S T R A C T Most patients with seropositive rheumatoid arthritis, and a variable but lesser percentage of normal subjects, have precipitating antibodies to a nuclear antigen, rheumatoid arthritis nuclear antigen, present in Epstein-Barr virus-infected human B lymphoblastoid cells. We have used a sensitive indirect immunofluorescence assay for antibody to rheumatoid arthritis nuclear antigen in a study of patients with infectious mononucleosis and healthy control subjects. Of 110 sera from normal, college-age cadets, 58 were from individuals without prior EpsteinBarr virus infection, as indicated by the lack of antibody to viral capsid antigen. All of these also lacked activity to rheumatoid arthritis nuclear antigen. 52 sera were positive for antibody to viral capsid antigen, and antibody to rheumatoid arthritis nuclear antigen was present in $26(50 \%)$ of these. In 67 sequential sera from 11 college-age students with infectious mononucleosis who became positive for antibody to rheumatoid arthritis nuclear antigen, only 2 were positive during the $1 \mathrm{mo}$. Thereafter the incidence and titers increased progressively through the lst yr after infection. This time-course resembled that for the development of antibody to Epstein-Barr nuclear antigen, another transformation antigen in Epstein-Barr virus-infected B lymphocytes. The development of positivity for both was much later than that of anti-
\end{abstract}

Received for publication 31 December 1979 and in revised form 14 February 1980. body to the structural viral capsid antigen, which in the current study was always positive by $1 \mathrm{wk}$. Thus, antibody to rheumatoid arthritis nuclear antigen is present in a large proportion of normal individuals and can now be clearly ascribed, from both in vivo and in vitro studies, to prior infection with Epstein-Barr virus.

\section{INTRODUCTION}

In vitro infection of $B$ lymphocytes with the EpsteinBarr virus $(\mathrm{EBV})^{1}$ results in the appearance of the Epstein-Barr nuclear antigen (EBNA) in the transformed B cell nuclei (1) and the later appearance of the viral capsid antigen (VCA) in Epstein-Barr viral particles (2). Alspaugh and co-workers have described another EBV-associated antigen, which appears in the nuclei of transformed B cells 20-30 d after in vitro EBV infection (3). They have designated it the rheumatoid arthritis nuclear antigen (RANA) because patients with seropositive rheumatoid arthritis have an increased frequency of precipitating antibody to it, as detected by Ouchterlony immunodiffusion (4). We recently demonstrated that anti-EBNA antibody titers were significantly higher in anti-RANA ${ }^{2}$-positive than

\footnotetext{
${ }^{1}$ Abbreviations used in this paper: EBNA, Epstein-Barr nuclear antigen; EBV, Epstein-Barr virus; IF, indirect immunofluorescence; IM, infectious mononucleosis; RANA, rheumatoid arthritis nuclear antigen; VCA, viral capsid antigen of the EBV.

"Anti-RANA was called "RAP” (RA precipitin) by Alspaugh and Tan, but for simplicity we refer to it as anti-RANA.
} 
negative sera, irrespective of whether or not they came from patients with rheumatoid arthritis or control subjects (5). These observations suggested a positive relationship between antibodies to EBNA and RANA in the normal population, and a subset of normals from which patients who subsequently develop rheumatoid arthritis may derive.

Natural in vivo EBV infection during adolescence or young adulthood causes infectious mononucleosis (IM) in about $50 \%$ of cases (6). Within 1-2 wk of disease onset, patients become seropositive for antiVCA (6). Anti-EBNA appears later, usually 1 mo or more after the onset of IM (7). Until now, the relationship of anti-RANA to in vivo EBV infection has not been defined. We undertook these studies to determine the frequency of anti-RANA in sera from normal individuals by the sensitive technique of indirect immunofluorescence (IF), to determine whether antiRANA only appears after EBV infection, and to compare the development of the anti-RANA response to that of anti-VCA and of anti-EBNA during the course of IM.

\section{METHODS}

Sera. These were obtained from three groups of subjects. Single serum samples were collected from 110 normal entering cadets at the U. S. Coast Guard Academy, New London, Conn., for studies of EBV infections in this population. 67 sera were obtained by Dr. Niederman from 11 undergraduates at Yale University, New Haven, Conn., as part of ongoing prospective studies of IM. These sera were collected serially at times ranging from $1 \mathrm{wk}$ to $5 \mathrm{yr}$ after onset of clinical IM and were used to determine the patterns of anti-VCA, anti-EBNA, and anti-RANA responses after primary EBV infection. The third group consisted of 59 patients with heterophile-positive and/or anti-VCA-positive IM from whom single serum samples were sent to Dr. Feorino at the Center for Disease Control, Atlanta, Ga., for EBV serology. All 59 sera were collected during the 1 mo after the onset of IM and were assayed for anti-RANA. All sera were aliquoted to minimize subsequent thaws and were stored at $-20^{\circ} \mathrm{C}$. Phosphate-buffered saline, $\mathrm{pH}$ 7.4, was used for serum dilutions.

Heterophile antibody assay. Sera from IM patients were assayed for heterophile antibodies by the method of Davidsohn and Lee (8).

Anti-VCA and anti-EBNA assays. Anti-VCA was measured by IF as described by Henle and Henle (2). Sera were considered positive if a 10 -fold dilution produced the typical diffuse cytoplasmic pattern of immunofluorescence.

Anti-EBNA was assayed similarly by the anticomplement IF method of Reedman and Klein (1) using the WI-L2 human B lymphoblastoid cell line as substrate, exactly as described (5). Sera were considered positive if a twofold dilution produced the typical, coarsely granular, nuclear immunofluorescence (1). Known positive and negative control sera were titered with each set of test sera and yielded reproducible titers.

Anti-RANA assay. Recent work in our laboratory has shown that human B lymphoblastoid cells maximally express RANA during the early "gap 1" phase of the cell replica-
TABLE I

Anti-RANA Frequencies* in Anti-VCA (-) and Anti-VCA (+) Normal Sera

\begin{tabular}{lccr}
\hline & \multirow{2}{*}{$\begin{array}{c}\text { Number } \\
\text { tested }\end{array}$} & Anti-RANA (+) \\
\cline { 3 - 4 } & 58 & 0 & Percentage \\
\hline Anti VCA (-) & 52 & 26 & $(0) \ddagger$ \\
Anti VCA (+) & $52) \ddagger$ \\
\hline
\end{tabular}

* By indirect immunofluorescence of 10 -fold diluted sera. $\ddagger$ Chi square $=35.2, P<10^{-8}$.

tion cycle. ${ }^{3}$ To assay RANA, the EBV-containing cell line WI-L2 (5) was therefore first synchronized into "gap l" by suspension of density-inhibited stationary phase cultures in fresh medium, as described by Lerner and Hodge (9). $4 \mathrm{~h}$ later, the cells were harvested, washed, and cytocentrifuged as for the anti-EBNA assay $(1,5)$. The preparations were then heat-fixed at $37^{\circ} \mathrm{C}$ for $15 \mathrm{~min}$. Using this substrate, antiRANA was detected by anti-immunoglobulin (Ig) IF of 10fold diluted sera, exactly as described by Alspaugh et al., (3) using rhodamine conjugated anti-human IgG (Bionetics, Laboratory Products, Litton Bionetics, Kensington, Md.) as the secondary antiserum. Sera were considered positive if a 10 -fold dilution produced the typical, finely granular, nuclear pattern of immunofluorescence.

Non-EBV-determined antinuclear antibodies. Preparations of the EBV-genome-negative $\mathrm{T}$ lymphoblastoid cell lines, CCRF-CEM (10), from the American Type Culture Collection (Rockville, Md.) were made as for WI-L2 to test antiEBNA-positive test sera and anti-RANA-positive test sera for non-EBV-determined antinuclear antibodies by anticomplement IF, and by indirect IF, respectively. All sera tested were negative.

Statistics. Anti-RANA frequencies were compared by the chi-square method with Yates' correction (11).

\section{RESULTS}

Prevalence of anti-RANA in anti-VCA-positive and negative persons. 110 healthy cadets were studied. 52 were anti-VCA-positive. Only 4 of these $(7.6 \%)$ had a history of IM, and the remaining 48 had thus undergone prior, clinically silent EBV infection. 26 of the $52(50 \%)$ were anti-RANA positive. Conversely, all of the 58 anti-VCA-negative cadets were also anti-RANA negative. The difference in anti-RANA frequencies is highly significant (Table I).

Times of appearance of anti-RANA, anti-EBNA, and anti-VCA after acute IM. 11 Yale University undergraduates, who were bled a total of 67 times after the onset of IM, each yielded at least one serum sample positive for anti-RANA. Fig. 1 shows the percentage of sera per time point that was anti-RANA positive

${ }^{3}$ Slovin, S. F., J. H. Vaughan, and D. A. Carson. Expression of Epstein-Barr-associated transformation antigens, EBNA and RANA, in synchronized cell cultures. Submitted for publication. 


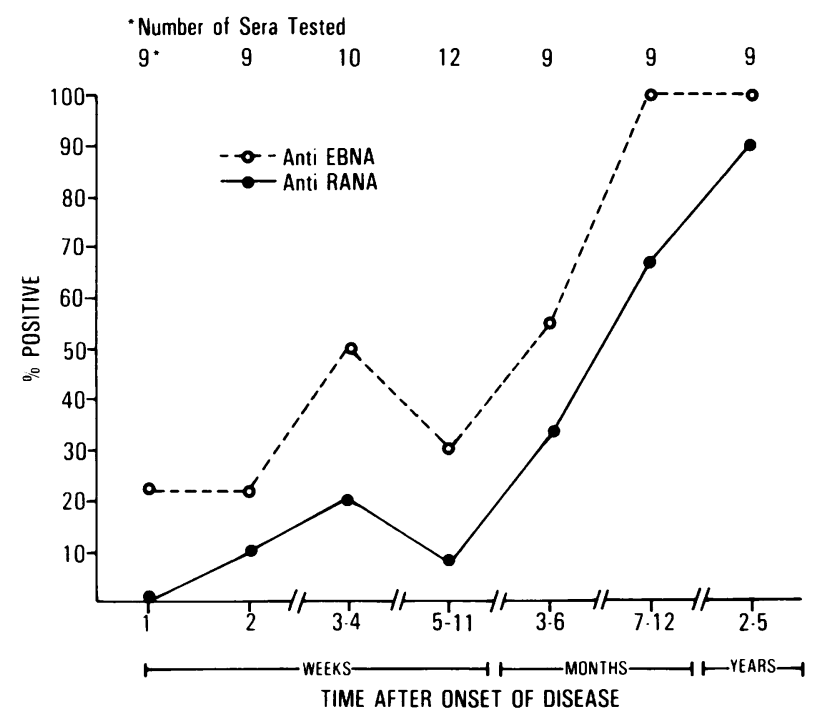

FIGURE 1 Times of appearance of anti-RANA and anti-EBNA after acute IM in Yale undergraduates. 67 sequential sera from 11 subjects were assayed for anti-RANA (O) by indirect IF of 10-fold diluted sera and for anti-EBNA $(O)$ by anticomplement IF of twofold diluted sera. Not shown: $100 \%$ of 1 wk sera were anti-VCA-positive by IF at a 10-fold dilution. and anti-EBNA positive. There were at least nine for each of seven time points up to $5 \mathrm{yr}$ after the onset of IM. All the sera at each time point were from different individuals except for the 12 sera collected at 5-12 wk, 2 of which were collected from the same subject at different times (one was positive and the other was negative for anti-RANA). The anti-RANA frequencies were $20 \%$ in the $3-4$ wk sera and $67 \%$ in the $7-12$ mo sera, respectively. By comparison, the anti-EBNA frequencies at the same time points were 50 and $100 \%$, respectively. In contrast, the frequency of anti-VCA (not depicted) reached $100 \%$ at $1 \mathrm{wk}$.

The 59 sera from the Center for Disease Control were obtained from $59 \mathrm{IM}$ patients during the 1 st mo of illness. Only seven (12\%) were positive for antiRANA, thus confirming the low frequencies of antiRANA in the Yale student sera during the same time interval.

Titers of anti-RANA and anti-EBNA after acute IM. 39 sequential sera from six of the Yale undergraduates were titered for anti-RANA and anti-EBNA (Fig. 2). For both antibodies, titers did not begin to rise until at least $40 \mathrm{~d}$ after the onset of IM, after which they rose nearly simultaneously and to comparable levels. The

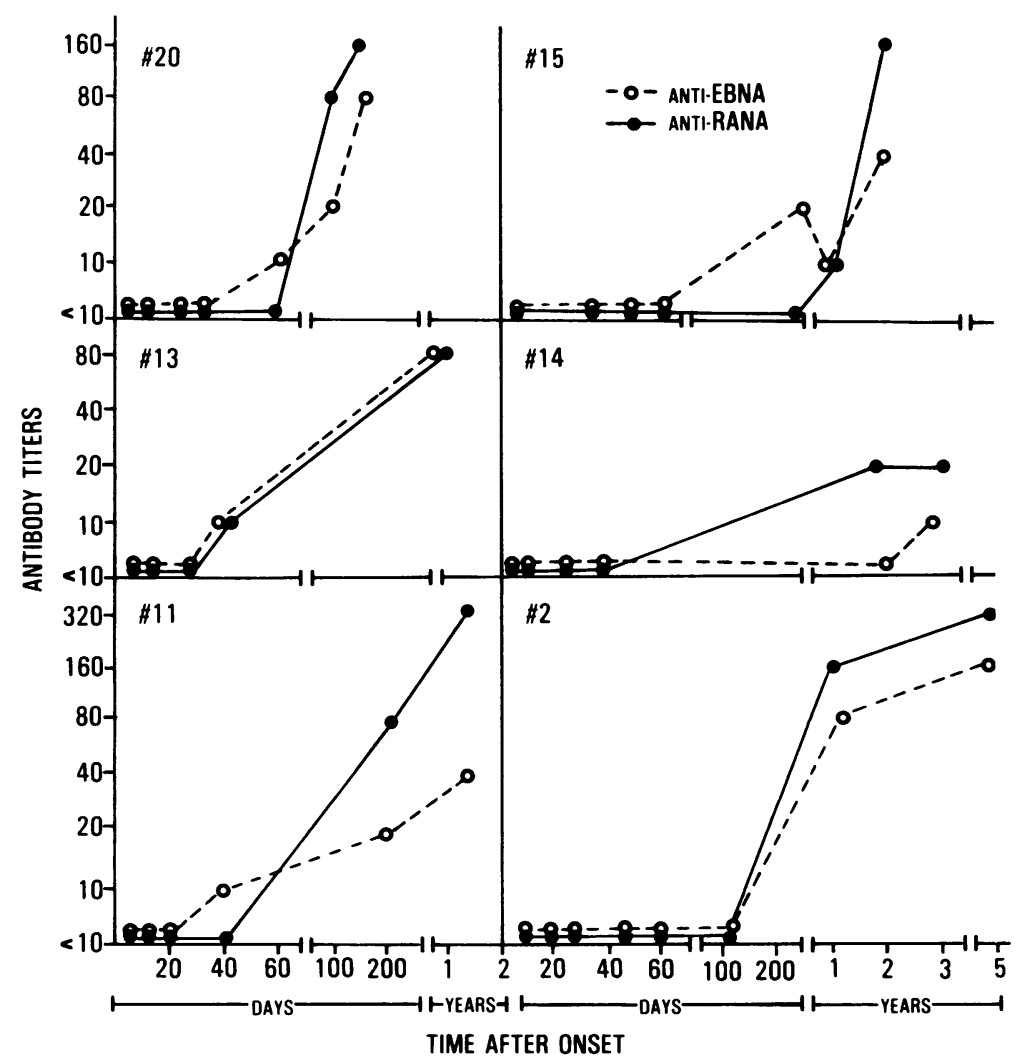

FIgURE 2 Titers of anti-RANA and anti-EBNA after acute IM in Yale undergraduates. 39 sequential sera from six subjects were assayed. Methods and symbols are as for Fig. 1. 
longest followup was achieved in cases 14 and 2 , in which titers of anti-RANA (case 14), and of both antiRANA and anti-EBNA (case 2), were not greatly different at 3 and $5 \mathrm{yr}$ from those at 1-2 yr.

\section{DISCUSSION}

These studies indicate that anti-RANA appears only after in vivo EBV infection (Table I), as was already known for anti-VCA (6) and anti-EBNA (7). This result is consistent with the observation that these antigens have been detected in vitro only after EBV infection of B cells (1-3). We have also shown that anti-RANA frequencies (Fig. 1) and titers (Fig. 2) parallel those of anti-EBNA, increasing significantly months after the onset of IM. They are distinct in their time-course from the much earlier-appearing anti-VCA. Titers of anti-RANA did not start to plateau until after $1 \mathrm{yr}$.

Several observations indicate that RANA and EBNA are different antigens. Sera have been found with antibodies exclusively to one and not to the other (3). RANA and EBNA have different solubilities in ammonium sulfate, and EBNA cannot be detected by immunofluorescence in transformed $B$ cells after treatment of the cells with deoxyribonuclease, while RANA is unaffected by such treatment $(12,13)$. RANA has a fine, granular appearance (3), whereas EBNA has a coarse, granular appearance (1). Recently, experiments with hybrids of EBV-transformed human B cells and rodent fibroblasts have shown that RANA and EBNA segregate into separate hybrid sublines, strongly suggesting that RANA and EBNA are distinct entities. ${ }^{4}$

In our previous studies of anti-RANA in normal adult individuals (5), we employed a more sensitive immunoprecipitation in gel than that used by Alspaugh and Tan (4) and consequently increased the percentage of anti-RANA positives in the normals to $25 \%$ from Alspaugh and Tan's $8 \%$. In the present study using immunofluorescence, we have found a $50 \%$ positivity in recently infected anti-VCA positive college-age students. As indicated by the time study in Fig. 2 , an investigation of older anti-VCA-positive adults would be expected to reveal an even higher percentage positive for anti-RANA, and a recent study in our laboratory of 72 normal adult sera has actually revealed a $75 \%$ figure.

In our previous study (5), it was intriguing that normal persons positive for anti-RANA precipitins also had higher titers of anti-EBNA than did normal persons negative for anti-RANA precipitins. In this respect,

+ Slovin, S. F., M. C. Glassy, M. A. Catalano, R. Curry, S. Ferrone, J. H. Vaughan, and D. A. Carson. Segregation of two Epstein-Barr-associated transformation antigens, EBNA and RANA, in human-rodent somatic cell hybrids. Submitted for publication. normal persons with anti-RANA precipitins resemble patients with seropositive rheumatoid arthritis, and therefore we have speculated that patients with seropositive rheumatoid arthritis may derive from that population of otherwise normal individuals who are hyperresponsive to RANA and EBNA. The hyperresponsiveness we observed did not include elevated levels of antibodies to several other herpes viruses, and probably not to VCA. The reasons for the selective hyperresponsiveness to RANA and EBNA are not known, but may conceivably lie in the fact that both RANA and EBNA are transformation antigens, whereas VCA and the other viral antigens we investigated in that study are virus structural antigens. As documented in the present study, the normal immune response to the transformation antigens follows distinctly different kinetics than does the normal immune response to viral structural antigens. Whether this is important in interpreting the rheumatoid arthritis data remains to be determined.

\section{ACKNOWLEDGMENTS}

We are indebted to the following people: the staff of the Yale University Health Service; Dr. Edward Blasser, Dr. Edward Shaskan, and the medical staff of the U. S. Coast Guard Academy Hospital; the physicians who sent sera to the Center for Disease Control; Sofia Freer and Dorreth Caplan for expert technical assistance; and Susan Lyons, Anna Milne, and Amy Deragon for expert secretarial work.

These studies were supported in part by research grants from the National Institutes of Health; AM 07144, AM 25443, AM 21175, RR 00833, RR 05514, AI 08731 and AI 14741; and by a grant from the Rheumatic Diseases Research Foundation.

\section{REFERENCES}

1. Reedman, B. M., and G. Klein. 1973. Cellular localization of an Epstein-Barr virus-associated complementfixing antigen in producer and nonproducer lymphoblastoid cell lines. Int. J. Cancer. 11: 499-520.

2. Henle, G., and W. Henle. 1966. Immunofluorescence in cells derived from Burkitt's lymphoma. J. Bacteriol. 91: $1248-1256$.

3. Alspaugh, M. A., F. C. Jensen, H. Rabin, and E. M. Tan. 1978. Lymphocytes transformed by the Epstein-Barr virus. Induction of nuclear antigen reactive with antibody in rheumatoid arthritis. J. Exp. Med. 147: 1018-1027.

4. Alspaugh, M. A., and E. M. Tan. 1976. Serum antibody in rheumatoid arthritis reactive with a cell-associated antigen. Arthritis Rheum. 19: 711-719.

5. Catalano, M. A., D. A. Carson, S. F. Slovin, D. D. Richman, and J. H. Vaughan. 1979. Antibodies to Epstein-Barr virus-determined antigens in normal subjects and in patients with seropositive rheumatoid arthritis. Proc. Natl. Acad. Sci. U. S. A. 76: 5825-5828.

6. Niederman, J. C., R. W. McCollum, G. Henle, and W. Henle. 1968. Infectious mononucleosis. Clinical manifestations in relation to $\mathrm{EB}$ virus antibodies. JAMA (J. Am. Med. Assoc.). 203: 205-209.

7. Henle, G., W. Henle, and C. A. Horwitz. 1974. Antibodies 
to Epstein-Barr virus-associated nuclear antigen in infectious mononucleosis. J. Infect. Dis. 130: 231-239.

8. Davidsohn, I., and C. L. Lee. 1964. Serologic diagnosis of infectious mononucleosis. Am. J. Clin. Pathol. 41: 115125.

9. Kaplan, J., T. C. Shope, and W. D. Peterson, Jr. 1974. Epstein-Barr virus-negative human malignant T-cell lines. J. Exp. Med. 139: 1070-1076.

10. Lerner, R. A., and L. D. Hodge. 1971. Gene expression in synchronized lymphocytes. Studies on the control of gene synthesis of immunoglobulin polypeptides. J. Cell Physiol. 77: 265-272.

11. Cohen, T. 1974. Statistics in Medicine. Little, Brown \& Co., Boston, Mass. 1st edition. 174-177.

12. Alspaugh, M. A., and E. M. Tan. 1975. Antibodies to cellular antigens in Sjogren's syndrome. J. Clin. Invest. 55: 1067-1073.

13. Baron, D., and J. L. Strominger. 1978. Partial purification and properties of the Epstein-Barr virus-associated nuclear antigen. J. Biol. Chem. 253: 2875-2881. 